\section{$\mathbf{m} / \mathbf{S}$}

médecine/sciences 1994; 10: 505-6

\title{
LE STROMA TUMORAL: UNE CIBLE POTENTIELLE DANS LE TRAITEMENT DES CANCERS
}

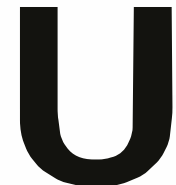

n des objectifs prioritaires de la recherche en cancérologie est d'appréhender les nombreux mécanismes qui contrôlent l'évolution des tumeurs vers des stades invasifs et métastatiques.

Les recherches sur la progression tumorale font l'objet de très nombreux travaux démontrant que les cellules malignes ont un génome instable. De nombreux réarrangements chromosomiques provoquent la perte ou l'activation de gènes par translocation. Les mutations ponctuelles ou l'amplification de gènes non altérés contribuent aussi à la disparition du contrôle de propriétés cellulaires, comme la prolifération ou l'acquisition et le maintien de l'état différencié. Les cellules cancéreuses ont souvent une origine clonale mais elles ne conservent pas nécessairement un phénotype et un génotype homogène au sein d'une même tumeur primaire. On considère cependant qu'une dominance clonale intervient au cours de la progression tumorale selon un principe néo-darwinien donnant ainsi naissance aux clones cellulaires les plus performants pour accomplir toutes les étapes du processus invasif et métastatique. On peut aussi considérer qu'une tumeur puisse garder une forte hétérogénéité et permettre à des cellules de phénotypes différents de progresser vers des phases plus malignes. Des données récentes, obtenues avec une lignée de carcinome vésical, montrent que l'on peut augmenter de façon très significative la tumorigenèse en introduisant, dans un inoculat injecté dans la souris, une faible proportion de cellules produisant un facteur de croissance. Aucune dominance clonale n'est observée dans la tumeur primaire ni dans les métastases ganglionnaires [1]. Le comportement de cette tumeur hétérogène rappelle celui décrit par Gurdon dans le mécanisme d'induction du mésoderme dorsal dans la lignée myogénique. Un ensemble de cellules embryonnaires bien délimité, soumis à une variété de signaux, répond de façon identique grâce au mécanisme de l'effet de communauté [2]

Les tumeurs ne sont pas seulement composées de cellules cancéreuses. Leur développement s'accompagne presque systématiquement de la formation d'un stroma. Jusque récemment, les recherches en oncologie moléculaire ont porté presque exclusivement sur les cellules cancéreuses, en faisant l'hypothèse que leur comportement autonome est responsable de l'évolution tumorale. Des travaux récents ont cependant attiré l'attention sur le rôle joué par le stroma dans le développement des tumeurs. Le stroma est composé de différents types cellulaires d'origine mésenchymateuse. Une majorité de cellules d'origine fibroblastique, souvent associées à une matrice extracellulaire extrêmement importante, adoptent un phénotype rappelant celui des cellules musculaires lisses. Grâce à une série de mar05 , France.

$\mathrm{m} / \mathrm{s} n^{\circ} 5$ vol. 10, mai 94 
queurs spécifiques du cytosquelette de la cellule musculaire lisse, il a été possible de mettre en évidence une forte hétérogénéité d'expression au sein de cette population dénommée myofibroblastes [3].

Des infiltrations de lymphocytes, de monocytes et de macrophages sont aussi très fréquemment observées. Les lymphocytes $\mathrm{T}$ intratumoraux (TIL) font d'ailleurs l'objet de recherches très poussées en vue d'application en immunothérapie. Enfin, les tumeurs ne pouvant se développer au-delà de quelques $\mathrm{mm}^{3}$ sans vascularisation, on observe la présence de nombreux vaisseaux dans le stroma péritumoral. Certains auteurs pensent, comme Folkman, que l'angiogenèse est certainement le mécanisme le plus important dans les phases précoces de la progression tumorale. La détermination semi-quantitative du nombre de vaisseaux peut, en effet, apporter dans certaines tumeurs un élément pronostique décisif [4].

Les deux articles de synthèse publiés dans ce numéro mettent l'accent sur une série de travaux récents montrant que le stroma des tumeurs peut jouer un rôle majeur dans la progression tumorale. L'article de B. Vandenbunder et al. (p.516 de ce numéro) analyse les mécanismes responsables de l'angiogenèse. De nombreux facteurs de croissance ont des propriétés angiogéniques, mais seuls le VEGF et ses récepteurs sont, à l'heure actuelle, de bons candidats in vivo. Tout récemment, une preuve supplémentaire de leur rôle critique dans l'angiogenèse a été apportée en inoculant des cellules d'une lignée de glioblastome produisant le VEGF avec des cellules productrices de particules rétrovirales codant pour une forme tronquée du récepteur Flk-1 du VEGF. L'expression de ce récepteur Flk-1, mutant dominant-négatif, par les cellules endothéliales infectées inhibe fortement la néovascularisation des tumeurs obtenues [5]. L'article de B. Vandenbunder et al. fait aussi le point sur des mécanismes de dégradation de la matrice extracellulaire facilitant la migration des cellules endothéliales. Contrairement aux résultats obtenus in vitro, il apparaît qu'in vivo, les cellules endothéliales ne produisent pas elles-mêmes les protéases mais seulement des inhibiteurs de la protéolyse comme les TIMP (tissue inhibitor of metalloproteinases) qui règleraient le niveau d'extension de l'angiogenèse. B. Vandenbunder et ses collaborateurs ont découvert que le facteur de transcription Ets-1 est exprimé chez l'embryon, dans les cellules endothéliales au cours de la vasculogenèse et de l'angiogenèse, et dans diverses populations cellulaires impliquées dans des remodelages tis sulaires et les migrations. Ce facteur de transcription n'est plus exprimé chez l'adulte, mais réapparaît de façon intense dans le mécanisme d'angiogenèse au cours de la réparation tissulaire et dans les tumeurs néovascularisées. De façon très intéressante, il s'exprime aussi dans une sous-population de cellules stromales étroitement associées aux cellules tumorales. Le facteur de transcription Ets-1 pourrait donc être impliqué comme régulateur du processus migratoire et invasif, en activant une batterie de gènes qui peuvent coder pour des protéases mais aussi des facteurs favorisant la mobilité cellulaire. Ets-1 est incontestablement une cible pour freiner le mécanisme de l'angiogenèse péritumorale.

L'article de C. Wolf et al. (p. 507 de ce numéro) réunit un ensemble de données nouvelles suggérant fortement que les cellules stromales, et particulièrement les myofibroblastes, jouent un rôle critique dans la progression tumorale. Il était communément admis que seules les cellules cancéreuses produisent les protéases et les glycanases nécessaires au franchissement de la lame basale et à leur pénétration dans le stroma. Grâce aux travaux récents basés sur l'hybridation in situ, il apparaît aujourd'hui que, dans une majorité des tumeurs, les protéases peuvent être aussi synthétisées par les fibroblastes entourant les cellules cancéreuses. Ainsi, dans l'adénocarcinome du colon, les cellules cancéreuses expriment-elles le récepteur de l'urokinase; cette enzyme est, ellemême, produite par les fibroblastes, alors que l'inhibiteur de l'activateur du plasminogène (PAI-1) est sécrété par les cellules endothéliales. Plusieurs métalloprotéases, dont les gélatinases, sont produites par les macrophages et les fibroblastes [6]. Dans les cancers de la peau, le stroma produit plusieurs métalloprotéases, dont la stromélysine de type I, cela jusqu'au stade carcinome squameux. L'évolution vers une forme plus grave de ces cancers (cancers à cellules fusiformes) est associée à une forte expression de cette protéase par les cellules tumorales [7].

Une nouvelle stromélysine (ST3), identifiée par Paul Basset et ses collaborateurs, fournit un exemple particulièrement démonstratif du rôle joué par le stroma des tumeurs. Cette enzyme n'est d'ailleurs pas spécifique des tumeurs car elle joue vraisemblablement un rôle important dans l'embryogenèse et dans la réparation tissulaire. Il est intéressant de noter que la ST3 est exprimée dans le stroma tumoral avant la phase invasive; à ce stade, elle pourrait participer au remodelage de cette composante tumorale en favorisant la prolifération et la migration des myofibroblastes et des cellules endothéliales.

Ces deux articles soulignent l'importance qu'il faut désormais accorder au stroma des tumeurs. Ce tissu complexe se développe en étroite interaction avec les cellules cancéreuses, en partie par le biais de facteurs diffusibles. Il joue donc un rôle capital dans la progression des tumeurs dont l'évolution ne peut dépendre exclusivement des cellules cancéreuses. Une meilleure connaissance des propriétés du stroma tumoral doit déboucher vers diverses stratégies thérapeutiques (antiangiogènes, inhibiteurs de protéases, cytostatiques). Certains composés sont déjà utilisés dans des essais cliniques 